\title{
Prevalence of seroadaptive behaviours of men who have sex with men, San Francisco, 2004
}

\author{
J M Snowden, ${ }^{1,2}$ H F Raymond, ${ }^{2}$ W McFarland ${ }^{2}$
}

${ }^{1}$ School of Public Health, University of California, Berkeley, California, USA; ${ }^{2}$ San Francisco Department of Public Health, San Francisco, California, USA

Correspondence to: Willi McFarland, HIV Epidemiology Section, San Francisco Department of Public Health, 25 Van Ness Avenue, Suite 500, San Francisco, CA 94102-6033, USA; willi_mcfarland@hotmail.com

Accepted 20 May 2009

\section{ABSTRACT}

Objectives: To define and measure the prevalence of HIV seroadaptive behaviours among men who have sex with men (MSM).

Methods: A community-based, cross-sectional sample of 1211 HIV negative and 251 HIV positive MSM was recruited in San Francisco in 2004 by time-location sampling. Seroadaptive behaviours were defined by enumerating and characterising all episodes of anal intercourse by partner type, partner HIV serostatus, sexual position and condom use for up to five partners in the preceding 6 months.

Results: Among HIV negative MSM, 37.6\% engaged in some form of apparent seroadaptive behaviour, predominantly pure serosorting (24.7\%), followed by seropositioning (5.9\%), condom serosorting (3.9\%) and negotiated safety (3.1\%). Among HIV positive men, $43.4 \%$ engaged in some form of seroadaptation, including pure serosorting (19.5\%), seropositioning (14.3\%) and condom serosorting (9.6\%). Consistent condom use was reported by $37.1 \%$ of HIV negative and $20.7 \%$ of HIV positive MSM.

Conclusions: In aggregate, seroadaptive behaviours appear to be the most common HIV prevention strategy adopted by MSM in San Francisco as of 2004. Surveillance and epidemiological studies need to precisely measure seroadaptive behaviours in order to gauge and track the true level of HIV risk in populations. Rigorous prevention research is needed to assess the efficacy of seroadaptive behaviours on individuals' risk and on the epidemic.

Studies from North America, Europe, Australia and Asia have reported increases in HIV incidence, sexually transmitted infections (STIs) or unprotected anal intercourse (UAI) among men who have sex with men (MSM) over the last several years. ${ }^{1-8}$ In some instances, increases in UAI or STI are accompanied by increases in HIV incidence or new HIV diagnoses. ${ }^{4-7}$ In other cases, increases in STI or UAI are not associated with increases in HIV infections. ${ }^{8-10}$

Serosorting is a phenomenon that has garnered attention in recent years and may explain some of the variations and apparent discrepancies in UAI, STI and new HIV infection. ${ }^{10-21}$ Broadly, serosorting is based on the simple idea that HIV transmission does not occur between persons of the same serostatus; however, in detail serosorting is complex. Among HIV positive people, there is the possibility that HIV transmission actually does occur (that is, superinfection) with implications for drug resistance and treatment options. ${ }^{22}$ Serosorting among HIV negative people carries the uncertainties that they may not have accurate knowledge of their own serostatus, their partners' serostatus and that they may test HIV antibody negative but may actually be in the highly infectious pre-seroconversion window period.

Definitions of serosorting used around the world are variable and nuanced by intentions, relationship status and specific sexual practices. For example, serosorting may occur at the level of selecting partners; that is, people choose to have only sex partners of the same HIV serostatus as themselves regardless of relationship type or sexual practices. Or, serosorting may occur at the level of condom negotiation; whereby persons dispense with condoms if they are of the same HIV serostatus, otherwise they use them if serodiscordant or in doubt. Other terminologies have been employed to distinguish particular practices that appear related to serosorting. Some researchers, notably in Australia, have defined negotiated safety as an agreement within a regular partnership to have UAI after both partners test HIV negative, twice 6 months apart, with mutual disclosure and consistently using condoms with other partners (for example, "talk, test, test, trust"), ${ }^{23}{ }^{24}$ and may also have a criterion that the other partners are also HIV negative. Strategic positioning or seropositioning describes UAI between known serodiscordant partners based on the rationale that HIV transmission is less likely to occur when the HIV positive person is in the receptive position and the HIV negative person is in the insertive position. ${ }^{12}{ }^{13}$ Given the confusion differing definitions of serosorting may engender, and given that MSM appear to have adopted a wide range of related practices with different degrees of risk reduction, other researchers have proposed an umbrella term of "seroadaptation" to encompass all the above behaviours and others remaining to be described. ${ }^{20}$

We concur with the need for a general, blanket term, such as seroadaptation, and for more precise definitions of related seroadaptive behaviours. The lack of clear definitions hinders precise measurement of risk for HIV acquisition and transmission and comparison of risk and preventive behaviours over time and between populations. During the 2004 round of the US National HIV Behavioral Surveillance (NHBS) for MSM, a survey coordinated in 25 state and local health departments by the Centers for Disease Control and Prevention (CDC), ${ }^{25-27}$ we added partner-by-partner questions in the local San Francisco instrument to the national core questionnaire that enabled distinguishing sexual practices according to respondents' and partners' HIV serostatus. The present study uses these supplemental questions to better define seroadaptation and to estimate the prevalence of several seroadaptive behaviours among MSM in San Francisco as of 2004. 


\section{METHODS}

\section{Study subjects, recruitment and sampling design}

We examined data collected from MSM who participated in the CDC-coordinated NHBS conducted in San Francisco. Details of the NHBS methods have been previously described. ${ }^{25-27}$ NHBS is designed as serial cross-sectional surveys in populations at high risk for HIV infection, rotating sequentially through MSM, IDUs and high risk heterosexuals in 25 cities in the USA. The primary aims of the NHBS are to measure HIV prevalence, related sexual and drug use behaviours, and exposure to prevention and care programmes. In the long term, NHBS will track changes in these indicators over time. The present study uses data from the first wave of NHBS, which recruited MSM through time-location sampling (TLS) in San Francisco between October 2003 and December 2004. ${ }^{27}$

The TLS methodology is used to recruit an approximated probability based sample in hard-to-reach populations throughout the world. ${ }^{28}$ TLS is based on the creation of a list of the of venues, days and time periods where and when the target population congregates to use as a sampling frame. In the present study, formative research was used to enumerate all gay-identified recruitment venues, which included bars, dance clubs, cruising areas, bookstores, gyms, social organisations, churches, street locations, and other venue types and the days and time periods of attendance. From this roster of all possible venue-day-time (VDT) periods, a random sample of VDT is drawn. At the VDT, the attendance of all potentially eligible subjects is enumerated by counting all individuals crossing a predetermined line or zone. Research staff intercept men entering the zone to determine eligibility. If eligible, men are invited to participate and informed consent is obtained. Intercepts are done consecutively without the choice of the recruiter until all staff are occupied. Once a staff member is available, consecutive intercepts and interviews resume. Thus, many more persons are enumerated as attending the venue than are intercepted. Sampling fractions for weights are determined using the enumerations. Participation rates are determined by the proportion of men determined eligible who complete the interview. Data are weighted according to the sample fraction recruited at the VDT and standard errors are adjusted for clustering. The methods have been applied in several previous surveys of MSM in San Francisco. ${ }^{4} 2930$

Eligibility criteria were male, age 18 years and older, being a resident of any of nine San Francisco Bay Area counties and being approached by the staff at the randomly selected VDT. Identifying as gay, bisexual or MSM was not an eligibility criterion in order to avoid excluding men who may not acknowledge male-male behaviour at the screening stage. At the analysis stage we included only MSM defined as either identifying as gay or bisexual or having had at least one male anal or oral sex partner in the past 12 months.

\section{Measures and analysis}

We present point prevalence and $95 \%$ confidence intervals (CIs) for several seroadaptive behaviours of HIV positive and HIV negative MSM. Seroadaptive behaviours were based on respondent's self-reported HIV serostatus, the reported serostatus of their partners and the sexual practices with each partner in the preceding 6 months collected anonymously on a hand-held computer. Seroadaptive constructs were created using measures present in the standardised NHBS national instrument plus the addition of a series of questions unique to the San Francisco NHBS. The national survey instrument asked men the date of their most recent HIV test and the result of that test. Sexual behaviours in the national instrument were aggregated across partner types (for example, main, casual, exchange, anonymous). The local San Francisco survey asked men questions on a partner-by-partner basis for up to five partners with whom they had sex in the last 6 months. If men had more than five partners in the 6 month period they were asked to report on the five partners they had sex with most frequently. For each partner, the participant was asked the partner's HIV serostatus, the partnership type (as above), the number of episodes of receptive anal intercourse, the number of episodes of unprotected receptive anal intercourse, the number of episodes of insertive anal intercourse and the number of episodes of unprotected insertive anal intercourse. The question pertaining to partner's HIV serostatus asked for only serostatus (positive, negative or unknown) and did not gather any information on how and to what level of certainty the respondent had ascertained his partner's serostatus nor whether they disclosed their serostatus to the partner.

From these data and drawing from the published literature on serosorting, we constructed hierarchical schema of seroadaptative behaviours for HIV negative and HIV positive MSM (fig 1 and 2 , respectively). These categories describe only the behavioural pattern over the 6 months preceding the interview; questions were not asked about intentions, certainty about partners' serostatus or longer term strategies adopted. Definitions were applied to people even if they had only one partner in the last 6 months with the exception of negotiated safety that required two or more partners. Also, with the exception of negotiated safety, which applied only to HIV negative men with a single main partner, we did not take into account the relationship type in our working definitions. The rationale was that, in the absence of direct measures, we did not make assumptions about intentions or levels of certainty about serostatus. Moreover, many participants reported multiple main partners in the 6 month period. We operationalised the following mutually exclusive categories or definitions.

\section{Abstainers}

Men who reported no anal sex partners in the last 6 months were categorised as abstainers. This category may include person engaging in sexual behaviours other than anal sex (for example, oral sex, mutual masturbation) or those who had no sex partners at all.

\section{$100 \%$ condom users}

This category comprised men who did have anal intercourse during the study period, but reported using condoms for all episodes of anal intercourse, for all partners, for both insertive and receptive positions. We did not consider abstaining from anal intercourse or $100 \%$ condom use as seroadaptive behaviours; that is, at least some UAI must occur to qualify as seroadaptation in our schema.

\section{Pure serosorters}

Men were considered pure serosorters if they had some UAI, but only had partners who were their same serostatus. That is, they were not considered pure serosorters if they used condoms $100 \%$ of the time with all partners nor if they had any partner of opposite or unknown serostatus regardless of whether they used condoms with them. The rationale of pure serosorting is that partner selection is based on HIV serostatus. 
Figure 1 Schema of seroadaptive behaviours of HIV negative men who have sex with men (MSM), San Francisco, 2004. Al, anal intercourse; UAl, unprotected anal intercourse; serodiscordant refers to partners of unknown or opposite serostatus.

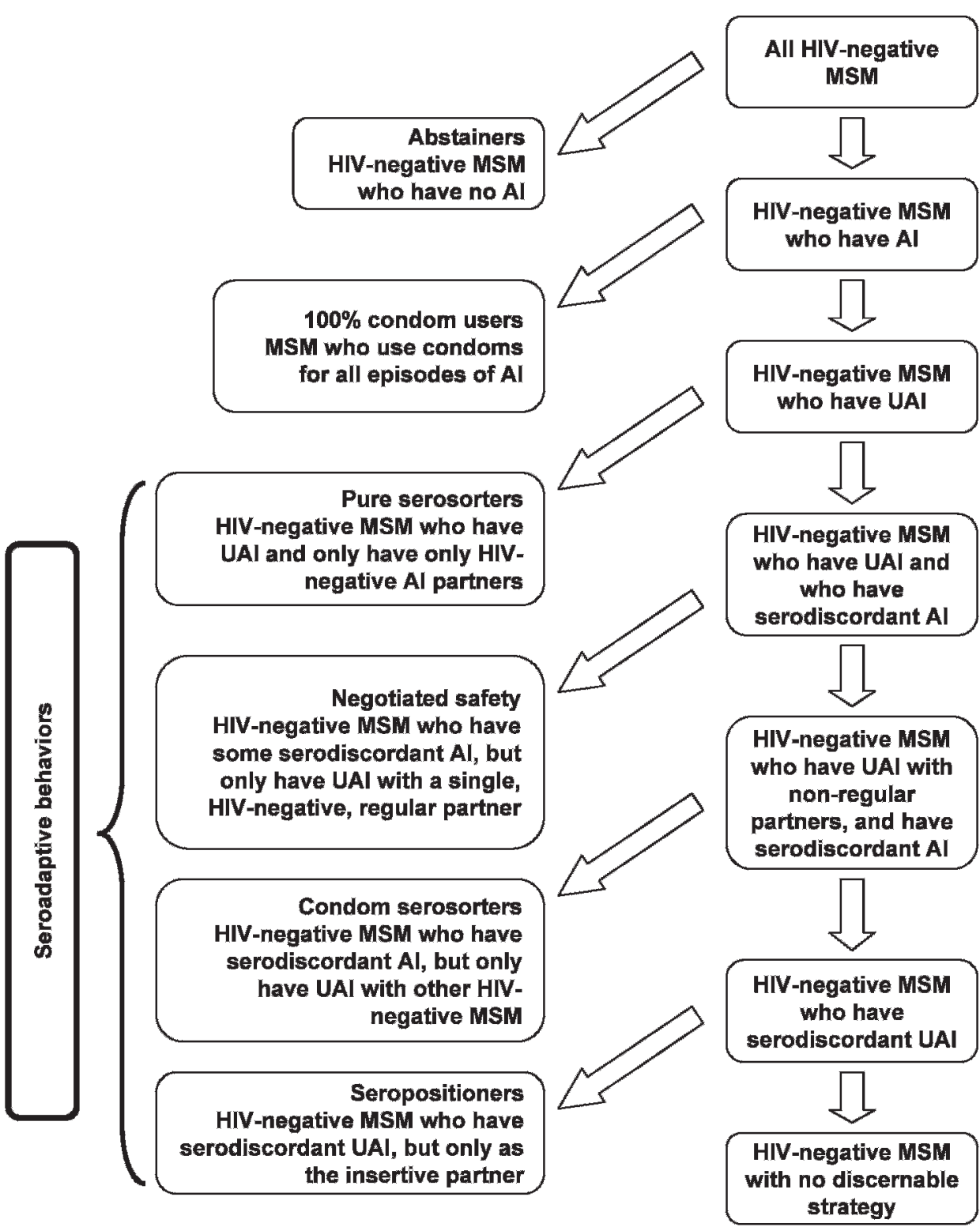

Negotiated safety

For HIV negative men only, we constructed a negotiated safety indicator as men who reported one main partner of HIV negative serostatus, with whom he had UAI and also reported non-main partners with whom he used condoms $100 \%$ of the time. HIV negative men whose behaviour matched this description, but who had had anal sex with exclusively HIV negative partners, were classified as pure serosorters, and were not considered to practice negotiated safety. We recognize that this operationalisation of negotiated safety falls short of previously published descriptions; ${ }^{23}{ }^{31}$ however, the questions necessary to meet all the requirements were not asked. Therefore, our definition may be a maximum level of potential negotiated safety as some, but not all, criteria were met.

\section{Condom serosorters}

Men were classified as condom serosorters if they did not fit into the above categories, had at least one partner of known serodiscordant or unknown serostatus (that is, potentially serodiscordant), but they only had UAI with known seroconcordant partners. In contrast to pure serosorting, the rationale for condom serosorting is that condom negotiation is based on HIV serostatus not partner selection.

\section{Seropositioners}

To be classified as a seropositioner, the respondent had to report UAI with a potentially HIV serodiscordant partner, but all episodes of potentially serodiscordant UAI were in the insertive position for HIV negative respondents and in the receptive position for HIV positive respondents. In keeping with the mutually exclusive nature of our seroadaptation categories, seropositioners did not meet the criteria of any of the above categories.

No discernable prevention strategy

This final grouping contains men who reported any anal intercourse but did not meet the criteria for any of the above categories. These men reported at least one episode of serodiscordant UAI in the insertive position for HIV positive men and in the receptive position for HIV negative men. 
Figure 2 Schema of seroadaptive behaviours of HIV positive men who have sex with men (MSM), San Francisco, 2004. Al, anal intercourse; UAl, unprotected anal intercourse; serodiscordant refers to partners of unknown or opposite serostatus.

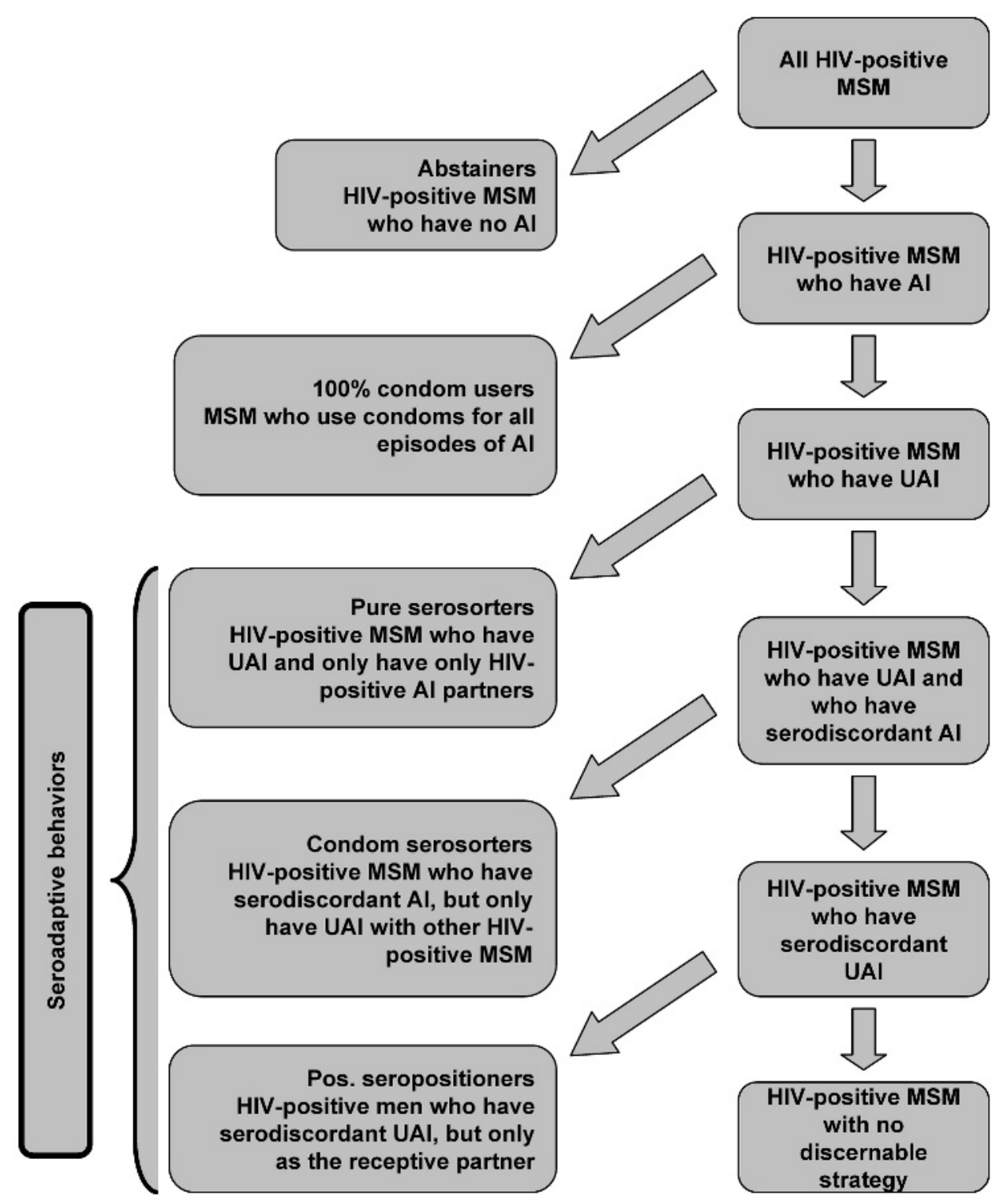

\section{Human subjects}

NHBS for 2004 was determined to be a non-research surveillance activity by both the CDC Institutional Review Board and University of California San Francisco's Committee on Human Research.

\section{RESULTS}

From November 2003 through December 2004, NHBS staff enumerated 44477 men in attendance at 169 randomly selected VDT in San Francisco and 3568 men were intercepted and screened for eligibility. Of the 2488 men who were eligible, 1764 $(70.9 \%)$ completed the behavioural survey. Of those, 1574 (89.2\%) identified as gay or bisexual or reported having at least one male sex partner in the past 12 months. Of these men, 1211 (76.9\%) self-reported as being HIV negative and 251 (15.9\%) reported being HIV positive. Overall, 112 (7.1\%) men reported not knowing their HIV serostatus, either by never having tested or not getting the results of their test. These men were excluded from further analyses based on the rationale that, at a minimum, purporting to know one's HIV serostatus is required in order to engage in seroadaptive behaviours. Overall, $17.7 \%$ had more than five partners; the analysis of seroadaptive behaviours included up to five partners per respondent using those with whom the respondent had sex with most often.

\section{HIV negative MSM}

Figure 3 shows the mutually exclusive categorisation of seroadaptive behaviours for HIV negative participants. Of the 1211 HIV negative MSM, 214 (17.7\%; 95\% CI 15.6 to $19.9 \%$ ) reported no anal intercourse in the last 6 months. A total of 449 HIV negative men (37.1\%; CI 34.3 to $39.9 \%$ ) reported $100 \%$ condom use with all partners for all episodes of anal intercourse- insertive and receptive. MSM reporting no anal intercourse or consistent condom use, together $54.7 \%$ (CI 51.9 to $57.6 \%$ ), would not meet any of our definitions of seroadaptation.

Of the HIV negative participants who had UAI with at least one sex partner, 299 (24.7\% of all HIV negative men; 95\% CI 22.3 to $27.2 \%$ ) were categorised as pure serosorters on the basis of reporting only partners of HIV negative serostatus. We identified 37 HIV negative men (3.1\% of all HIV negative men; CI 2.2 to $4.2 \%$ ) who met our operational criteria for negotiated 
Figure 3 Prevalence of seroadaptive behaviours of HIV negative men who have sex with men (MSM) in the last 6 months, San Francisco, 2004. Al, anal intercourse; UAl, unprotected anal intercourse; serodiscordant refers to partners of unknown or opposite serostatus.

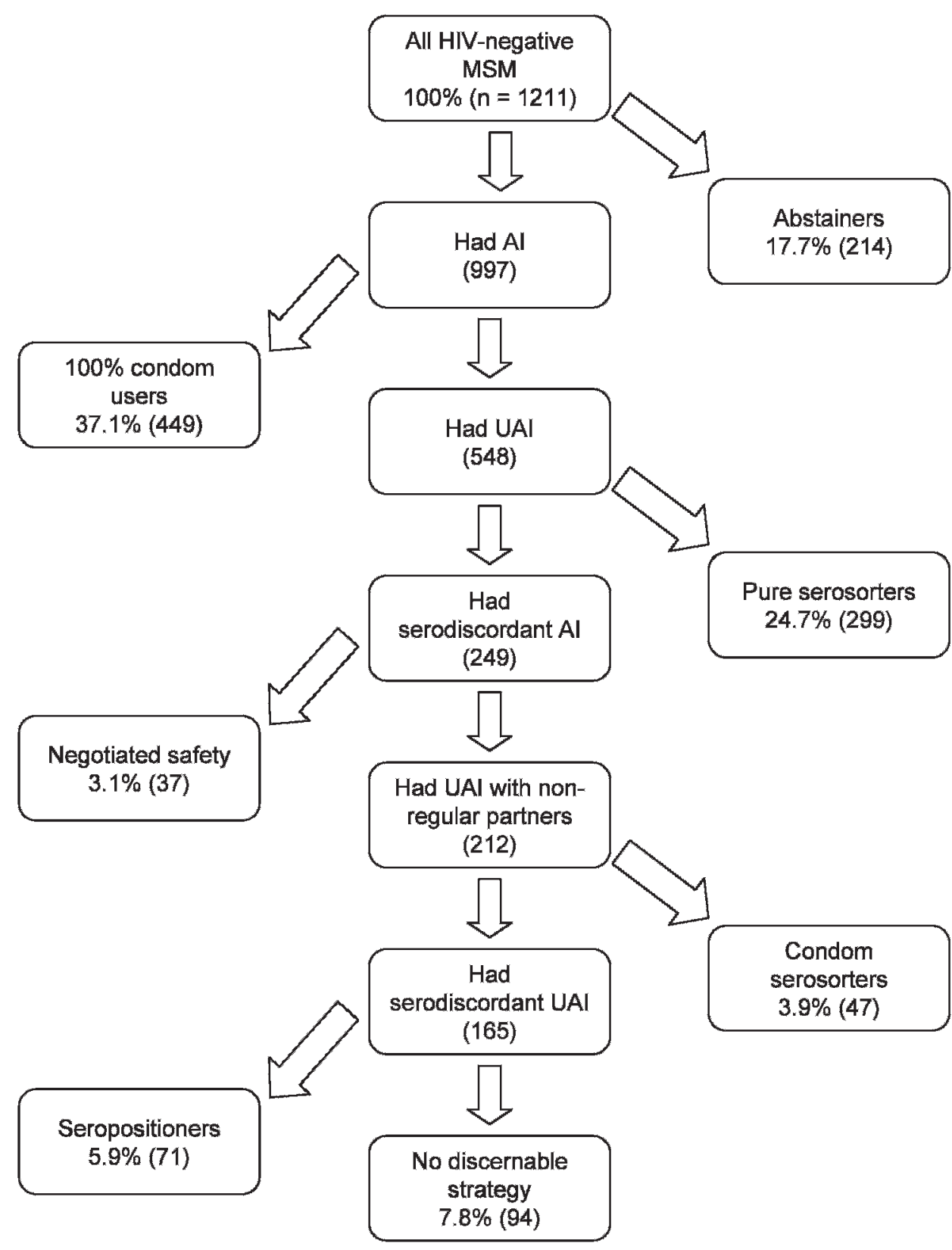

safety; that is, they reported UAI exclusively with their HIV negative regular partner and consistent condom use with all other partners regardless of those partner's HIV serostatus. The definition of condom serosorting (that is, any episode of UAI with a HIV negative partner, but consistent condom use with HIV positive or unknown serostatus partners) was met by 47 men (3.9\% of all HIV negative men; $95 \%$ CI 2.9 to $5.1 \%$ ). For the remaining HIV negative men not falling into any of the aforementioned categories, 71 men $(5.9 \%$ of all HIV negative men; $95 \%$ CI 4.6 to $7.3 \%$ ) met the criteria as seropositioners (that is, all episodes of UAI with partners of HIV positive or unknown serostatus were insertive).

The remaining 94 men (7.8\% of all HIV negative participants; $95 \%$ CI 6.3 to $9.4 \%$ ) could not be categorised into any seroadaptive category. These HIV negative men reported having receptive UAI with partners of HIV positive or unknown serostatus.

\section{HIV positive MSM}

Figure 4 illustrates the seroadaptive behaviours of HIV positive participants. Of $251 \mathrm{HIV}$ positive participants, 46 (18.3\%; 95\% CI 13.7 to $23.7 \%$ ) reported no anal intercourse in the last 6 months, while 52 reported $100 \%$ condom use for episodes of anal intercourse $(20.7 \%$; $95 \%$ CI 15.9 to $26.2 \%$ ).

The remaining MSM $(61.0 \%$ of the HIV positive men in the study; $95 \%$ CI 54.6 to $67.0 \%$ ) were assessed for seroadaptive behaviours. We identified 49 men $(19.5 \%$ of all HIV positive participants; $95 \%$ CI 14.8 to $25.0 \%$ ) as meeting the criterion for pure serosorters, reporting only HIV positive sex partners. The 24 HIV positive participants reporting some HIV negative or unknown serostatus sex partners, but only UAI with their seroconcordant partners, were classified as condom serosorters (9.6\% of all HIV positive men; 95\% CI 6.2 to 13.9\%). Altogether, 36 men (14.3\% of all HIV positive participants; 95\% CI 10.3 to $19.3 \%$ ) met the criteria for seropositioners (that is, all episodes 
Figure 4 Prevalence of seroadaptive behaviours of HIV positive men who have sex with men (MSM) in the last 6 months, San Francisco, 2004. Al, anal intercourse; UAl, unprotected anal intercourse; serodiscordant refers to partners of unknown or opposite serostatus.

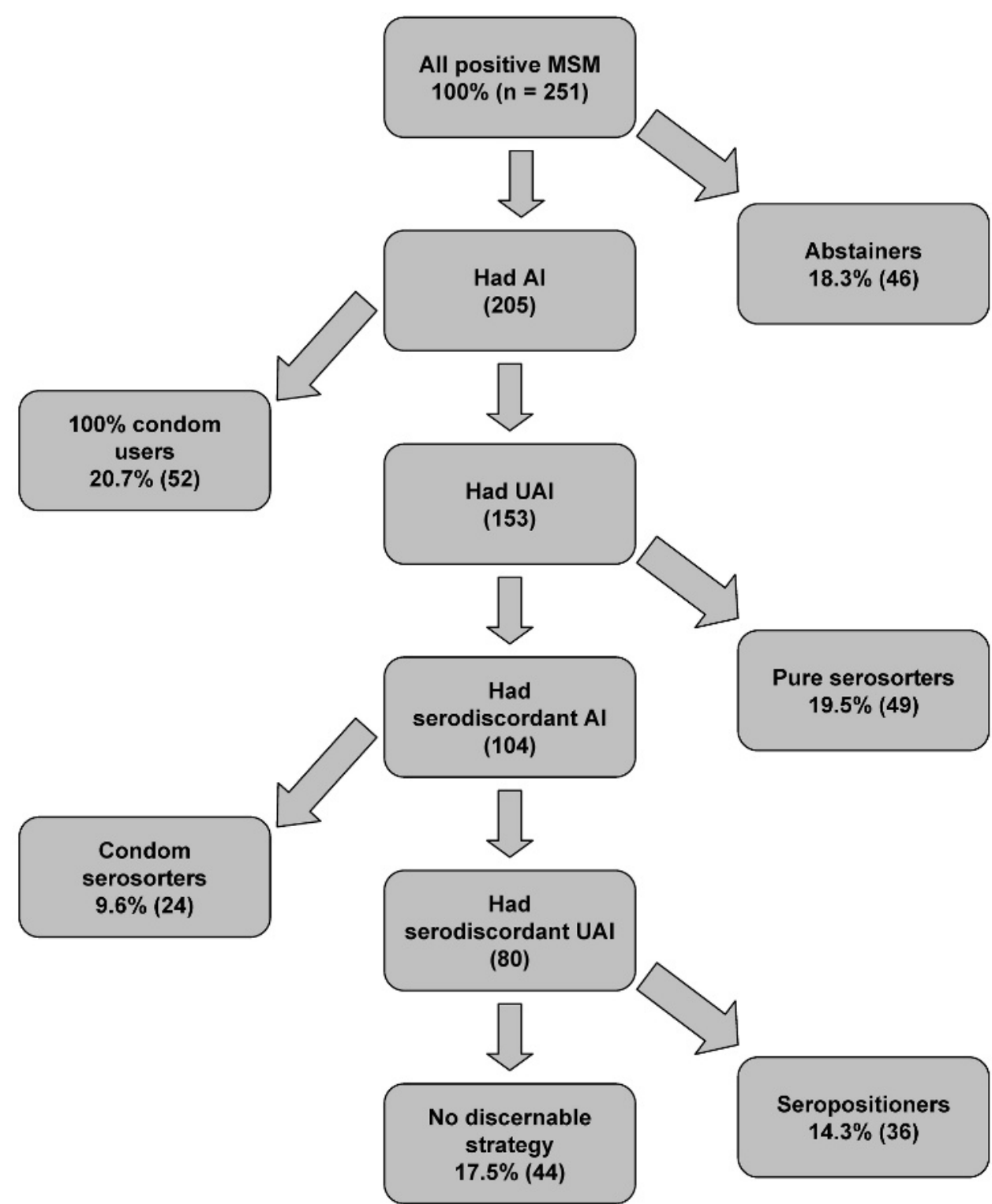

of UAI with partners of HIV negative or unknown serostatus were receptive).

After considering all the above categories, there remained 44 HIV positive men (17.5\% of all HIV positive men; 95\% CI 13.0 to $22.8 \%$ ) whose sexual behaviour indicated no discernable HIV prevention or seroadaptive strategy. These HIV positive men engaged in insertive UAI with partners of HIV negative or unknown serostatus.

Comparing the above figures between HIV negative and HIV positive MSM, HIV negative men were more likely to practice $100 \%$ condom use than HIV positive men $(p<0.001)$, while HIV positive MSM were more likely to be condom serosorters $(p<0.001)$ and seropositioners $(p<0.001)$ than HIV negative MSM. HIV negative MSM were more likely to be pure serosorters at a borderline significant level $(p=0.08)$. Comparing across all seroadaptive strategies, HIV positive MSM were more likely to practice any seroadaptive strategy than HIV negative MSM at a borderline significance level $(p=0.079)$.

\section{DISCUSSION}

Using definitions guided by the literature and analysing episodes-based, partner-by-partner data on sexual behaviour in the preceding 6 months, we estimated the prevalence of several seroadaptive behaviours in a large community based sample of MSM. Among HIV negative MSM, 37.6\% engaged in some form of seroadaptive behaviour, predominantly pure serosorting $(24.7 \%)$, followed by seropositioning $(5.9 \%)$, condom serosorting $(3.9 \%)$ and negotiated safety (3.1\%). The figure was higher among HIV positive men: $43.4 \%$ overall with the most common being pure serosorting (19.5\%), followed by seropositioning $(14.3 \%)$ and condom serosorting (9.6\%). In aggregate, seroadaptation was more prevalent than consistent condom use among both HIV negative and HIV positive MSM (37.1\% and $20.7 \%$, respectively) and, therefore, the most common HIV prevention strategy adopted by MSM in San Francisco in 2004. Taken another way, seroadaptation appeared to fill a large middle ground of sexual harm reduction between safer practices such as abstaining from anal intercourse and consistent condom use and the riskier practices of receptive UAI with an unknown or HIV positive partner by HIV negative MSM (7.8\%) and insertive UAI with an unknown or HIV negative partner by HIV positive MSM (17.5\%).

Our data enhanced the understanding of the rising trend in overall UAI with falling trends in potentially serodiscordant 
UAI among MSM observed in the STOP AIDS Project data from 1998 to $2004 .{ }^{10}$ Concurrent data showing rising incidence of STIs and level HIV incidence lead to the interpretation that serosorting was stabilising or reversing a previous rise in HIV incidence among MSM in San Francisco. ${ }^{4}$ The current data from a separate study break down the types of seroadaptive behaviours present among MSM in 2004. It is disheartening that the STOP AIDS Project data collected since 2004 have shown an increase in potentially serodiscordant UAI (from 4\% in 1998 to $18 \%$ in 2007 among HIV negative MSM, and from $21 \%$ in 1998 to $32 \%$ in 2007 among HIV positive MSM) suggesting an erosion of at least pure serosorting and condom serosorting in the subsequent 3 years. ${ }^{32}$ Where measured, trends in seroadaptive behaviours among MSM elsewhere appear mixed. For example, serosorting was reported to be increasing among both HIV negative and HIV positive MSM attending STI clinics in Seattle from 2001 to $2007,{ }^{21}$ but with no evidence of an increase among HIV negative MSM in London despite a doubling among HIV positive MSM from 1998-2005. ${ }^{17}$

A consequence of our findings and the work of others among MSM worldwide is that it may no longer be accurate or useful to track trends in sexual risk without being able to discriminate between the various seroadaptive behaviours. Common measures, such as overall frequency of condom use with all partners, with certain partner types, condom use at last sex or even by partners aggregated by HIV serostatus, ${ }^{25-27}{ }^{33}$ will fall far short of being true indicators of preventive or risky behaviour. Behavioural surveillance, epidemiological surveys and sexual health research studies will need to record, at a minimum, each person's HIV serostatus, each partner's HIV serostatus, relationship type, a count of the episodes of sex in each position and a count of the episodes when condoms were used. We recognise that the approach requires a higher level of complexity than is typical in most surveys. However, challenges to the ease and flow of such instruments may be partially addressed by new computer-based technologies that automate skip patterns and identify inconsistencies. We found that the addition of our partner-by-partner episode count approach added approximately 5 minutes time to the standard NHBS core questionnaire-a difference that might effectively disappear with the removal of the behavioural questions aggregated across partners and partner types and those focused only on the last episode of sex.

Nonetheless, we recognise that our delineation between the seroadaptive behaviours reported here also fall short of measuring true preventive behaviour. First and most significantly, our operationalisation of recent practices did not capture the intentions behind them. It is likely that substantial proportions of respondents fell within our definitions not because they consciously adopted a particular seroadaptive strategy, but because of the relationship they currently find themselves in, certain preferences in sexual practices, a 6-month period that did not reflect a long-term strategy or other circumstances. For example, one may assume that some reporting no anal intercourse in the preceding 6 months did not deliberately abstain. A similar limitation pertains to those with only one partner in the time period. We also were unable to capture the behaviours with partners numbering above five per person, which amounted to $17.7 \%$ of subjects. Nonetheless, we captured all episodes of anal intercourse for the vast majority of respondents (82.3\%) and, for those who reported more than five partners, the partners included were those with whom they had sex with the most. This implies that our data cover the largest proportion of all anal intercourse episodes possible in the preceding 6 months. We also did not ask the level of certainty with which participants knew their own or their partners' HIV serostatus, whether they mutually disclosed, deduced in some other way or only guessed. Therefore, there is no way to verify the accuracy of the seroadaptive behaviours described here. Because our definition of seroadaptation is predicated on knowing one's own serostatus, we have not analysed the behavioural patterns among men of unknown serostatus $(7.1 \%)$ - a group who are crucial to an understanding of HIV transmission and risk behaviour among MSM.

There is also uncertainty in the hierarchy of risk we devised. For example, we assumed that no anal sex was less risky than protected anal sex, which is less risky than unprotected sex with someone presumed HIV negative and so on in order to exclusively assign participants to one category or strategy. Seroadaptive behaviours are likely to be very contextual and fluid, changing between types, and not necessarily adhered to exclusively. In our goal of attempting to estimate the point prevalence of several different types of seroadaptive behaviours, we recognise the trade-off in forcing categories to be mutually exclusive and the more protean realities of sexual behaviour.

In retrospect, we missed the opportunity to record other potential types of seroadaptive behaviours. For HIV positive men and their partners, antiretroviral therapy (ART) can be a factor in assessment of risk and sexual decision-making. ${ }^{34}{ }^{35}$ Similarly, an HIV positive person's viral load has been demonstrated to be related to the probability of transmission ${ }^{35}$ and to inform sexual decision-making and, thus, should also be considered a seroadaptive strategy. ${ }^{1134}{ }^{36}$ Recent policies and debate emanating from Switzerland may increase awareness and adoption of a seroadaptive behaviour of engaging in UAI with HIV serodiscordant partners when adherent to ART and viral load is suppressed. ${ }^{37} 38$ Other potential seroadaptive behaviours we did not measure include exclusive oral sex, oral sex with potentially HIV serodiscordant partners and withdrawal before ejaculation. ${ }^{39} 40$ We may also be unaware of other seroadaptive strategies currently prevalent among MSM. Future research will need to keep abreast of these community generated prevention strategies that are likely to change over time.

However, the main challenge to future research is determining which of these seroadaptive behaviours are effective or how they can be made effective. At least one prevention trial demonstrated a reduced risk of transmission from HIV infected MSM through an increase in the proportion of partners who were also HIV positive. ${ }^{41}$ However, other investigators have pointed to the potential increase in HIV transmission that may occur through UAI between people and within networks of people who wrongly assume they are seroconcordant and via acute infection when people would test antibody negative. ${ }^{42}{ }^{43} \mathrm{~A}$ prospective cohort study of HIV negative MSM in Sydney, Australia, offered evidence of differing levels of risk associated

\section{Key messages}

- Seroadaptive behaviours were the leading prevention approach of men who have sex with men (MSM) in San Francisco in 2004 , reported by $37.6 \%$ of HIV negative and $43.4 \%$ of HIV positive men.

- Surveillance and epidemiological studies need to measure seroadaptive behaviours in order to gauge and track the true level of HIV risk in populations.

- Prevention research is needed to assess the efficacy of seroadaptive behaviours on individuals' risk and on the epidemic. 
with various seroadaptive behaviours, with some increasing risk of HIV infection and others having no apparent effect. ${ }^{44}$ Additional longitudinal studies are required to assess the intentions with respect to seroadaptation before measuring subsequent behaviour in order to establish evidence that they are consciously adopted prevention strategies and not post-hoc rationalisations for having engaged in risky sex. Longitudinal studies are also needed to evaluate how well people can adhere to the various seroadaptive strategies over time. While prevalence estimates may gauge which seroadaptive behaviours are most commonly practiced, more detailed measures are needed to assess which are the most appealing to whom, how people implement them and which factors predict successful or unsuccessful adherence. Most difficult of all will be assessing if seroadaptation, implemented in its wide array of practices, is contributing to the ongoing and potentially rising HIV transmission among MSM or if it is part of a solution to stabilise, reduce and ultimately halt the epidemic.

Funding: National HIV Behavioural Surveillance is funded by the Division of HIV/AIDS Prevention, Centers for Disease Control and Prevention (U62/CCU923549).

\section{Competing interests: None.}

Contributors: JMS conducted the main analysis, provided the primary interpretation of results and wrote the first draft of the manuscript. HFR provided primary oversight to the implementation of the study, including data collection and data management, and contributed to the analysis, interpretation and writing of the manuscript. WM provided overall scientific oversight to the study, including the design and conceptualisation of the research question, and made the final manuscript edits.

Provenance and peer review: Not commissioned; externally peer reviewed.

\section{REFERENCES}

1. Calzavara L, Burchell AN, Major C, et al. Increases in HIV incidence among men who have sex with men undergoing repeat diagnostic HIV testing in Ontario, Canada. AIDS 2002:16:1655-61.

2. Dodds JP, Nardone A, Mercey DE, et al. Increase in high risk sexual behaviour among homosexual men, London 1996-8: cross sectional, questionnaire study. BMJ 2000;320:1510-11.

3. Centers for Disease Control and Prevention (CDC). HIV prevalence among populations of men who have sex with men-Thailand, 2003 and 2005. MMWR 2006;55:844-8.

4. Katz MH, Schwarcz SK, Kellogg TA, et al. Impact of highly active antiretroviral treatment on HIV seroincidence among men who have sex with men in San Francisco. Am J Public Health 2002;92:388-94.

5. Stolte IG, Dukers NH, de Wit JB, et al. Increases in sexually transmitted infections among homosexual men in Amsterdam in relation to HAART. Sex Transm Dis 2001;77:184-6.

6. Dukers NH, Spaargaren J, Geskus RB, et al. HIV incidence on the increase among homosexual men attending an Amsterdam sexually transmitted disease clinic: using a novel approach for detecting recent infections. AIDS 2002;16:F19-24.

7. Ma X, Zhang 0 , He X, et al. Trends in prevalence of HIV, syphilis, hepatitis C, hepatitis $B$, and sexual risk behavior among men who have sex with men. Results of 3 consecutive respondent-driven sampling surveys in Beijing, 2004 through 2006. $J$ Acquir Immune Defic Syndr 2007;45:581-7.

8. Middleton MG, Grulich AE, McDonald AM, et al. Could sexually transmissible infections be contributing to the increase in HIV infections among men who have sex with men in Australia? Sex Health 2008;5:131-40.

9. van der Bij AK, Stolte IG, Coutinho RA, et al. Increase of sexually transmitted infections, but not HIV, among young homosexual men in Amsterdam: are STIs still reliable markers for HIV transmission? Sex Transm Infect 2005;81:34-7.

10. Truong $\mathbf{H - H}$, Kellogg T, Klausner JD, et al. Increases in sexually transmitted infections and sexual risk behavior without a concurrent increase in HIV incidence among men who have sex with men in San Francisco: a suggestion of HIV serosorting? Sex Transm Infect 2006;82:461-6.

11. Suarez TP, Kelly JA, Pinkerton SD, et al. Influence of a partner's HIV serostatus, use of highly active antiretroviral therapy, and viral load on perceptions of sexual risk behavior in a community sample of men who have sex with men. J Acquir Immune Defic Syndr 2001;28:471-7.

12. van de Ven $\mathbf{P}$, Kippax $\mathrm{S}$, Crawford $\mathrm{J}$, et al. In a minority of gay men, sexual risk practice indicates strategic positioning for perceived risk reduction rather than unbridled sex. AIDS Care 2002;14:471-80.

13. Parsons JT, Schrimshaw EW, Wolitski RJ, et al. Sexual harm reduction practices of HIV-seropositive gay and bisexual men: serosorting, strategic positioning, and withdrawal before ejaculation. AIDS 2005;19:513-25.
14. Mao L, Crawford JM, Hospers HJ, et al. "Serosorting" in casual anal sex of HIVnegative gay men is noteworthy and is increasing in Sydney, Australia. AIDS 2006;20:1204-6.

15. Xia 0, Molitor F, Osmond DH, et al. Knowledge of sexual partner's HIV serostatus and serosorting practices in a California population-based sample of men who have sex with men. AIDS 2006;20:2081-9.

16. Osmond DH, Pollack LM, Paul JP, et al. Changes in prevalence of HIV infection and sexual risk behavior in men who have sex with men in San Francisco: 19972000. Am J Public Health 2007;97:1677-83.

17. Elford J, Bolding G, Sherr L, et al. No evidence of an increase in serosorting with casual partners among HIV-negative gay men in London, 1998-2005. AIDS 2007:21:243-5

18. van der Bij AK, Kolander ME, de Vries $\mathrm{HJ}$, et al. Condom use rather than serosorting explains differences in HIV incidence among men who have sex with men. J Acquir Immune Defic Sydr 2007:45:574-80.

19. Eaton LA, Kalichman SC, Cain DN, et al. Serosorting sexual partners and risk for HIV among men who have sex with men. Am J Prev Med 2007;33:479-85.

20. Le Talec J, Jablonski 0. Seroadaptation instead of serosorting: a broader concept and a more precise process model. http://www.thewarning.info/article. php?id article $=0249$ (accessed 11 June 2009).

21. Golden MR, Stekler J, Hughes JP, et al. HIV serosorting in men who have sex with men: is it safe? JAIDS 2008;49:212-18.

22. Blish CA, Dogan OC, Derby NR, et al. Human immunodeficiency virus type 1 superinfection occurs despite relatively robust neutralizing antibody responses. J Viral 2008;82:12094-103.

23. Victoria AIDS Council, Gay Men's Health Crisis website. http://www.vicaids asn.au/content/ContentPageasp?PagelD $=79$

24. Kippax S, Crawford J, Davis M, et al. Sustaining safe sex: a longitudinal study of a sample of homosexual men. AIDS 1993;7:257-63.

25. Gallagher KM, Sullivan PS, Lansky A, et al. Behavioral surveillance among people at risk for HIV infection in the U.S.: the National Behavioral Surveillance System. Public Health Reports 2007;122:32-38.

26. MacKellar D, Gallagher KM, Finlayson T, et al. Surveillance of HIV risk and prevention behaviors of men who have sex with men - a national application of venue based, time-space sampling. Public Health Reports 2007;122:39-47.

27. Sanchez T, Finlayson T, Drake A, et al. Human immunodeficiency virus (HIV) risk, prevention, and testing behaviors - United States, National HIV Behavioral Surveillance System: men who have sex with men, November 2003-April 2005. MMWR Surveillance Summaries 2006;55:1-16.

28. Magnani R, Sabin K, Saidel T, et al. Review of sampling hard-to-reach and hidden populations for HIV surveillance. AIDS 2005;19:S67-72.

29. Katz MH, McFarland W, Guillin V, et al. Continuing high prevalence of HIV and risk behaviors with men: The Young Men's Survey in San Francisco Bay Area in 1992-93 and 1994-95. J Acquir Immune Defic Syndr 1998;19:178-81.

30. Valleroy LA, MacKellar DA, Karon JM, et al. High HIV prevalence and associated risks among adolescent and young adult men who have sex with men in 7 US metropolitan areas in 1994 through 1998. JAMA 2000;284:198-204.

31. Crawford JM, Rodden P, Kippax S, et al. Negotiated safety and other agreements between men in relationships: risk practice redefined. Int J STD AIDS 2001:12:164-70.

32. Scheer S, Kellogg T, Klausner J, et al. HIV is hyper-endemic among men who have sex with men in San Francisco: Ten-year trends in HIV incidence, HIV prevalence, sexually transmitted infections, and sexual risk behavior. Sex Transm Infect 2008:84:493-8.

33. UNAIDS. National AIDS Program Guide to Monitoring and Evaluation. http://data. unaids.org/Publications/IRC-pub05/jc427-mon ev-full en.pdf (accessed 11 June 2009).

34. Ostrow DE, Fox KJ, Chmiel JS, et al. Attitudes towards highly active antiretroviral therapy are associated with sexual risk taking among HIV-infected and uninfected homosexual men. AIDS 2002;16:775-80.

35. Dukers NH, Goudsmit J, de Wit JB, et al. Sexual risk behaviour relates to the virological and immunological improvements during highly active antiretroviral therapy in HIV-1 infection. AIDS 2001;15:369-78.

36. Prestage G, Mao L, Kippax S, et al. Use of viral load to negotiate condom use among gay men in Sydney, Australia. AIDS Behav 2009

37. Quinn TC, Wawer MJ, Sewankambo N, et al. Viral load and heterosexual transmission of human immunodeficiency virus type 1. Rakai Project Study Group. N Engl J Med 2000:34:921-9.

38. http://www.swissinfo.ch/eng/search/Result.html?sitesect $=882 ধ y=$ stধsid $=8685522$

39. Page-Shafer K, Dilley J, McFarland W, et al. Risk of HIV infection attributable to oral sex among men who have sex with men and in the population of men who have sex with men. AIDS 2002;16:2350-2.

40. Richters J, Knox S, Crawford J, et al. Condom use and "withdrawal": explore gay men's practice of anal intercourse. Int J STD AIDS 2000;11:96-104.

41. Morin S, Shade SB, Steward WT, et al. A behavioral intervention reduces HIV transmission risk by promoting sustained serosorting practices among HIV-infected men who have sex with men. J Acquir Immune Defic Syndr 2008.

42. Butler DM, Smith DM. Serosorting can potentially increase HIV transmission. AIDS 2007:21:1218-20.

43. Pinkerton SD. Acute HIV infection increases the dangers of serosorting. Am J Prev Med 2008;35:184.

44. Jin F, Crawford J, Prestage GP, et al. Unprotected anal intercourse, risk reduction behaviours, and subsequent HIV infection in a cohort of homosexual men. AIDS 2009:23:243-52. 